\title{
Global patterns of body size evolution in squamate reptiles are not driven by climate
}

\author{
Alex Slavenko $^{1}$ (D) | Anat Feldman ${ }^{2}$ | Allen Allison $^{3}$ | Aaron M. Bauer ${ }^{4}$ | \\ Monika Böhm ${ }^{5}$ | Laurent Chirio ${ }^{2}$ | Guarino R. Colli ${ }^{6}$ | Indraneil Das ${ }^{7}$ | \\ Tiffany M. Doan ${ }^{8}$ | Matthew LeBreton ${ }^{9}$ | Marcio Martins ${ }^{10}$ | Danny Meirte ${ }^{11}$ | \\ Zoltán T. Nagy ${ }^{12}$ | Cristiano de C. Nogueira ${ }^{10}$ | Olivier S. G. Pauwels ${ }^{13}$ | \\ Daniel Pincheira-Donoso $^{14}$ (D) | Uri Roll ${ }^{15}$ | Philipp Wagner ${ }^{4,16}$ | Yuezhao Wang ${ }^{17}$ | \\ Shai Meiri ${ }^{1,18}$
}

\footnotetext{
${ }^{1}$ School of Zoology, Tel Aviv University, TelAviv, Israel

${ }^{2}$ Independent scholar

${ }^{3}$ Hawaii Biological Survey, 4 Bishop Museum, Honolulu, Hawaii

${ }^{4}$ Department of Biology, Villanova University, Villanova, Pennsylvania

${ }^{5}$ Institute of Zoology, Zoological Society of London, London, United Kingdom

${ }^{6}$ Departamento de Zoologia, Universidade de Brasília, Brasília, Brazil

${ }^{7}$ Institute of Biodiversity and Environmental Conservation, Universiti Malaysia Sarawak, Kota Samarahan, Malaysia

${ }^{8}$ Division of Natural Sciences, New College of Florida, Sarasota, Florida

${ }^{9}$ Mosaic (Environment, Health, Data,

Technology), Yaoundé, Cameroon

${ }^{10}$ Departamento de Ecologia, Instituto de Biociências, Universidade de São Paulo, São Paulo, Brazil

${ }^{11}$ Royal Museum for Central Africa, Tervuren, Belgium

${ }^{12}$ Joint Experimental Molecular Unit, Royal Belgian Institute of Natural Sciences, Brussels, Belgium

${ }^{13}$ Department of Recent Vertebrates, Royal Belgian Institute of Natural Sciences, Brussels, Belgium

${ }^{14}$ Department of Biosciences, School of Science and Technology, Nottingham Trent University, Nottingham, United Kingdom

${ }^{15}$ Mitrani Department of Desert Ecology, The Jacob Blaustein Institutes for Desert Research, Ben-Gurion University, Midreshet Ben-Gurion, Israel

${ }^{16}$ Allwetterzoo, Münster, Germany
}

\begin{abstract}
Aim: Variation in body size across animal species underlies most ecological and evolutionary processes shaping local- and large-scale patterns of biodiversity. For well over a century, climatic factors have been regarded as primary sources of natural selection on animal body size, and hypotheses such as Bergmann's rule (the increase of body size with decreasing temperature) have dominated discussions. However, evidence for consistent climatic effects, especially among ectotherms, remains equivocal. Here, we test a range of key hypotheses on climate-driven size evolution in squamate reptiles across several spatial and phylogenetic scales.

Location: Global.

Time period: Extant.

Major taxa studied: Squamates (lizards and snakes).

Methods: We quantified the role of temperature, precipitation, seasonality and net primary productivity as drivers of body mass across ca. $95 \%$ of extant squamate species (9,733 spp.). We ran spatial autoregressive models of phylogenetically corrected median mass per equal-area grid cell. We ran models globally, across separate continents and for major squamate clades independently. We also performed specieslevel analyses using phylogenetic generalized least square models and linear regressions of independent contrasts of sister species.

Results: Our analyses failed to identify consistent spatial patterns in body size as a function of our climatic predictors. Nearly all continent- and family-level models differed from one another, and species-level models had low explanatory power.

Main conclusions: The global distribution of body mass among living squamates varies independently from the variation in multiple components of climate. Our study, the largest in spatial and taxonomic scale conducted to date, reveals that there is little support for a universal, consistent mechanism of climate-driven size evolution within squamates.
\end{abstract}


${ }^{17}$ Chengdu Institute of Biology, Chinese Academy of Sciences, Chengdu, China

${ }^{18}$ Steinhardt Museum of Natural History, Tel Aviv University, Tel-Aviv, Israel

\section{Correspondence}

Alex Slavenko, School of Zoology, Tel Aviv University, Tel-Aviv 6997801, Israel.

Email: slavenko@mail.tau.ac.il

Editor: Brody Sandel

\section{KEYWORDS}

Bergmann's rule, body mass, body size, ectotherms, phylogenetic comparative analyses, reptiles, size clines, spatial analyses

\section{1 | INTRODUCTION}

Climate is traditionally considered a primary source of natural selection underlying the evolution of spatial, ecological and phylogenetic variation in animal body sizes. Given that most ecological and evolutionary processes operating among and within species are strongly influenced by body size (Peters, 1983), the identification of predictable relationships between size and geography has offered a key to elucidate the emergence of local- and large-scale patterns of biodiversity (e.g., Gillooly, Brown, West, Savage, \& Charnov, 2001; Siemann, Tilman, \& Haarstad, 1996; Slavenko, Tallowin, Itescu, Raia, \& Meiri, 2016; Woodward et al., 2005). Remarkably, this principle pre-dates the theory of evolution by natural selection itself Bergmann's (1847) seminal work suggested that body size among closely related mammal and bird species tends to increase towards colder geographical regions (James, 1970). Such spatial body size gradients have been found to be prevalent in endotherms, at both the intraspecific (Ashton, Tracy, \& de Queiroz, 2000; James, 1970; Meiri \& Dayan, 2003; Rensch, 1938; cf. Riemer, Gurlanick, \& White, 2018) and the interspecific (Blackburn \& Hawkins, 2004; Olson et al., 2009; Torres-Romero, Morales-Castilla, \& Olalla-Tárraga, 2016) level. In contrast, decades of research conducted on a wide range of ectothermic organisms have uncovered mixed support for climate-driven size clines at either the intraspecific (Adams \& Church, 2008; Ashton \& Feldman, 2003; Pincheira-Donoso, 2010; Pincheira-Donoso \& Meiri, 2013; Zamora-Camacho, Reguera, \& Moreno-Rueda, 2014) or the interspecific (Feldman \& Meiri, 2014; Olalla-Tárraga \& Rodríguez, 2007; Olalla-Tárraga, Rodríguez, \& Hawkins, 2006; PincheiraDonoso, Hodgson, \& Tregenza, 2008; Rodrigues, Olalla-Tárraga, Iverson, \& Diniz-Filho, 2018; Slavenko \& Meiri, 2015; Terribile, Olalla-Tárraga, Diniz-Filho, \& Rodríguez, 2009; Vinarski, 2014) level.

The lack of consistency in the attempts to identify prevalent drivers of body size evolution in ectotherms may be partly attributable to the lack of applicability of the heat-related mechanism (i.e., Bergmann's original explanation) to ectotherms (Meiri, 2011; Pincheira-Donoso et al., 2008; Slavenko \& Meiri, 2015). Bergmann (1847) posited that reduced surface area-to-volume ratio in larger animals benefits heat conservation in colder climates, a mechanism sometimes known as the "heat conservation hypothesis". However, ectotherms produce negligible amounts of metabolic heat, and reduced surface areato-volume ratios might result in less efficient thermoregulation in cold climates owing to slower heating rates. Therefore, a trade-off exists between heat gain (more efficient in smaller ectotherms; Carothers, Fox, Marquet, \& Jaksic, 1997) and retention (more efficient in large ectotherms; Zamora-Camacho et al., 2014). Thus, large body size in colder climates is predicted to compromise the need to achieve optimal body temperatures to initiate basic fitness-related activities in the first place (Pincheira-Donoso et al., 2008).

Alternative mechanisms for climate-driven body size clines may be more applicable to ectotherms. The "heat balance hypothesis" (Olalla-Tárraga et al., 2006) predicts that thermoconformers exhibit a reverse pattern to the one predicted by Bergmann's rule (i.e., smaller bodies at lower temperatures because of the effect of body size on heating rates). The "water availability hypothesis" (Ashton, 2002) suggests that large sizes, hence small surface area-to-volume ratios, are beneficial in conserving water in dry habitats (especially for animals with permeable skins, such as amphibians). Therefore, large size is predicted to be selected for in arid climates. The "starvation resistance hypothesis" (Boyce, 1979; Lindsey, 1966) and the "seasonality hypothesis" (Mousseau, 1997; Van Voorhies, 1996) both posit that seasonality drives size clines. The former suggests that large size is selected for in seasonal environments, because it allows for accumulation of food reserves to survive periods of food scarcity. The latter suggests that short growing seasons in highly seasonal climates lead to maturation at smaller size. The "primary productivity hypothesis" (Rosenzweig, 1968; Yom-Tov \& Geffen, 2006) suggests that increased productivity allows for the evolution of larger body sizes, which can be maintained by the abundance of available food (Huston \& Wolverton, 2011). These hypotheses are not mutually exclusive, and the different putative climatic drivers of size evolution covary across space.

We addressed a range of core hypotheses on the relationship between climate and body size globally across squamates, the largest order of land vertebrates (ca. 10,350 species; Uetz, Freed, \& Hošek, 2018). Squamates are found on all continents except Antarctica. Their distribution patterns differ considerably from other land vertebrate groups, showing increased affinity for hot, arid regions (Roll et al., 2017). However, most studies on climatic size clines in squamates have been conducted on species from temperate regions (e.g., Ashton \& Feldman, 2003; Olalla-Tárraga et al., 2006; Pincheira-Donoso, Tregenza, \& Hodgson, 2007). Therefore, the more limited scale of existing studies is unlikely to be representative of squamates, either phylogenetically (i.e., many families are not represented there) or geographically (i.e., the 